\title{
FAUNA DE COLEOPTERA LAMELLICORNIA DE LA ZONA CAÑERA DEL INGENIO DE ATENCINGO, PUEBLA, MÉXICO
}

\author{
Agustín ARAgÓN-GARCíA, ${ }^{1,4}$ Miguel Ángel MORÓN, ${ }^{2}$ Miguel Ángel \\ DAMIÁN-HUATO, ${ }^{1,4}$ Jesús Francisco LÓPEZ-OLGUÍN, ${ }^{1,4}$ ERIKA PATRICIA \\ PINSON-RINCÓN ${ }^{3,4}$ \& JosÉ NELSON PÉREZ-QUINTANILLA ${ }^{3,4}$ \\ ${ }^{1}$ Departamento de Agroecología y Ambiente. Instituto de Ciencias. Benemérita Universidad Autónoma \\ de Puebla. 14 sur 6301, C.U. 72570 Puebla, Puebla, México. Cuerpo Académico Ciencias Ambientales \\ y Agricultura.<agustin.aragon@correo.buap.mx> \\ ${ }^{2}$ Instituto de Ecología, Xalapa, Veracruz, Apartado Postal 63, Código Postal 91000, México \\ ${ }^{3}$ Facultad de Ciencias Agrícolas, Campus IV, Universidad Autónoma de Chiapas. Cuerpo Académico \\ de Protección Vegetal. \\ ${ }^{4}$ Red Nacional de Productividad y Calidad de Alimentos Agrícolas, PROMEP-SEP.
}

Aragón-García, A., M. Á. Morón, M. Á. Damián-Huato, J. F. López-Olguín, E. P. Pinson-Rincón \& J. N. Pérez-Quintanilla. 2012. Fauna de Coleoptera Lamellicornia de la zona cañera del ingenio de Atencingo, Puebla, México. Acta Zoológica Mexicana (n. s.), 28(1): 161-171.

RESUMEN. Con la finalidad de evaluar la riqueza de escarabajos (Scarabaeoidea) de la zona cañera de Atencingo al sur del estado de Puebla, se realizaron capturas durante 2000 al 2003, utilizando una trampa de luz tipo pantalla, provista con una lámpara de luz de vapor de mercurio de 120 watts, así como colecta directa bajo el alumbrado público y sobre follaje de árboles y arbustos. Se recolectaron un total de 1253 especímenes que representan a cuatro familias, seis subfamilias, 11 tribus, y 23 especies de los géneros Cotinis, Cyclocephala, Digitonthophagus, Diplotaxis, Epectinaspis, Ligyrus, Paranomala, Phileurus, Phyllophaga, Polyphylla, Ptichopus, y Strategus. El género mejor representado fue Phyllophaga con cinco especies. Entre las especies más abundantes se encuentran Cyclocephala lunulata y Phyllophaga brevidens con 534 y 539 ejemplares cada una. Se incluye una clave para identificar las especies encontradas.

Palabras clave: Cetoniidae, Melolonthidae, Passalidae, Scarabaeidae, caña de azúcar.

Aragón-García, A., M. Á. Morón, M. Á. Damián-Huato, J. F. López-Olguín, E. P. Pinson-Rincón \& J. N. Pérez-Quintanilla. 2012. Coleoptera Lamellicornia fauna of the sugar cane area of the sugar plantation of Atencingo, Puebla, Mexico. Acta Zoológica Mexicana (n. s.), 28(1): 161-171.

ABSTRACT. With the purpose of evaluating the abundance of beetles (Scarabaeoidea) of the sugar cane area of Atencingo southward of the state of Puebla, captures were carried out during the period of 2000 to 2003 by using a screen-type light trap provided with a 120 watts mercury vapor light lamp, as well as direct collection under the public illumination and on tree and bush foliage. A total of 1253 speci-

Recibido: 11/05/2011; aceptado: 06/09/2011. 
mens representing four families, six subfamilies, 11 tribes and 23 species of the genera Cotinis, Cyclocephala, Digitonthophagus, Diplotaxis, Epectinaspis, Ligyrus, Paranomala, Phileurus, Phyllophaga, Polyphylla, Ptichopus and Strategus were collected. Best represented genus was Phyllophaga with five species. Most abundant species were Cyclocephala lunulata and Phyllophaga brevidens, each with 534 and 539 specimens, respectively. A key to the 23 species is included.

Key words: Cetoniidae, Melolonthidae, Passalidae, Scarabaeidae, sugar-cane.

\section{INTRODUCCIÓN}

En México se han registrado poco más de 1700 especies de Scarabaeoidea incluidas en diez familias (Morón 2003, 2010), y las larvas de alrededor de 810 especies son edafícolas mostrando hábitos rizófagos, saprófagos o facultativos, por lo que no todas están asociadas con las plantas. Sin embargo, los miembros de los géneros Phyllophaga, Macrodactylus, y Paranomala representan algunos de los insectos subterráneos más conocidos por los agricultores, que pueden ocasionar pérdidas significativas en cultivos agrícolas con gran importancia en el país (Morón 1983, 2010).

Durante las investigaciones efectuadas entre 1996 y 2008 en diferentes ambientes característicos del estado de Puebla se ha confirmado la presencia de 264 especies de escarabajos incluidas en 88 géneros de 9 familias, cifras equivalentes al 15\% de las especies y al 44\% de los géneros de estos grupos a escala nacional (Aragón et al. 2010). De estas se han registrado 9 géneros y 41 especies de Melolonthidae y Cetoniidae que se encuentran asociadas a los cultivos de alfalfa, amaranto, arroz, cacahuate, café, calabaza, caña de azúcar, cebolla, jamaica, haba, frijol, cempasúchil, nopal, papa, rábano, sorgo, tomate y zanahoria (Aragón \& Morón 1998). Tal diversidad es alta en comparación con otros estados, y evidencia la complejidad de los ambientes característicos de la entidad. Es posible que cuando menos existan representantes de otras 50 especies que se han citado de Hidalgo, Morelos, Oaxaca y Veracruz, además de otras hasta ahora no descubiertas en los valles áridos, bosques aislados y zonas agrícolas.

Existen en México algunos coleópteros que por los daños que causan al cultivo de la caña de azúcar han sido considerados como plagas importantes en varios ingenios del país. Estos insectos son conocidos en el medio agrícola nacional con los nombres de "gallina ciega" o "nixticuil", "mayatito del tallo" y "mayate rinoceronte" (Melolonthidae); "picudos del tallo", "picudos del tronco" (Curculionidae) y "gusano de alambre" (Elateridae) (Aragón \& Morón 2000).

Debido a la importancia que revisten las plagas de la caña de azúcar y dado que en la parte suroeste del estado de Puebla, donde las condiciones son propicias para dicho cultivo, se tiene poca información sobre la fauna de coleópteros escarabaeoideos, se consideró oportuno llevar a cabo la presente investigación para evaluar la riqueza especifica de los escarabajos (Scarabaeoidea) en la región cañera de Atencingo, Puebla. 


\section{MATERIALES Y MÉTODOS.}

Zona de estudio. La zona cañera se encuentra localizada en la parte suroeste del estado de Puebla, en el municipio de Chietla, dentro del Valle de lzúcar de Matamoros. Sus coordenadas geográficas son 18' 26' $00^{\prime \prime}$ y 18' 36' 00" de latitud norte y 98' 31 ' 24" y 98' 42' 36" de longitud oeste presentando una altitud entre los 1080 y 1280 msnm (Fig. 1) (Anónimo 2005). El clima de la zona es semicálido subhúmedo con lluvias en verano y sequía notable en invierno, con una precipitación anual menor de

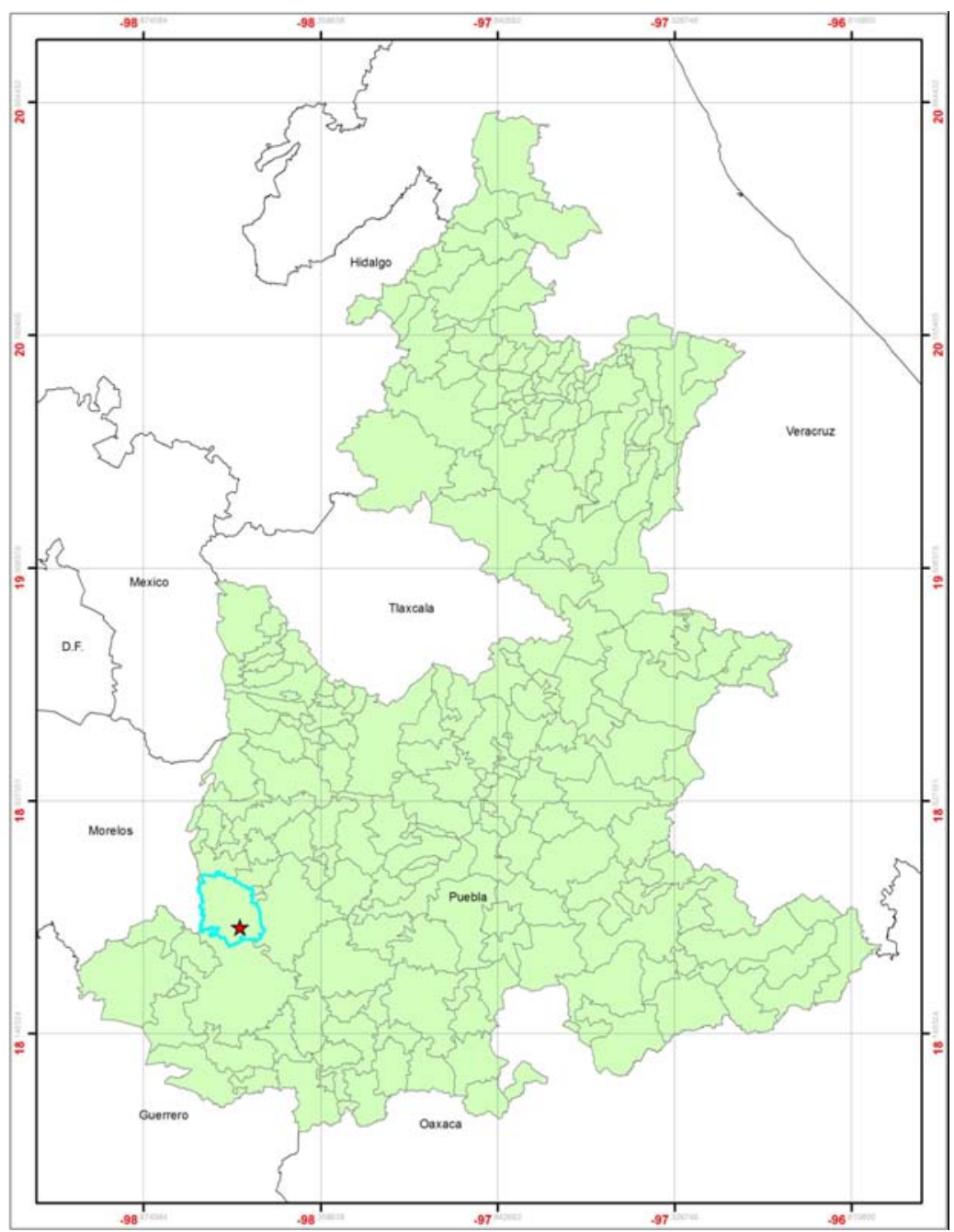

Figura 1. Localización geográfica de la zona cañera de Atencingo, Puebla. 
$800 \mathrm{~mm}$, la temperatura media anual oscila entre 22 y $26{ }^{\circ} \mathrm{C}$ (Anónimo 2008) por lo cual queda ubicada dentro del tipo de clima Aw" o (w) (I') g (según Koppen, modificado por García 1973).

Los suelos de la región son muy variados, pudiéndose identificar cinco grupos de suelos; Vertisoles que son suelos de textura arcillosa y pesada que se agrietan notablemente cuando se secan; Litosoles que son suelos de menos de $10 \mathrm{~cm}$. de espesor sobre roca o tepetate; Regosoles o suelos formados por material suelto que no sea aluvial reciente, son pobres en nutrientes y en ocasiones infértiles y Rendzinas que son suelos ricos en materia orgánica y nutrientes y Feozem que son suelos adecuados a cultivos y que toleran excesos de agua (Anónimo 2005).

La vegetación original que presentaba la zona corresponde a un bosque tropical bajo caducifolio asociado con vegetación secundaría arbustiva o arbórea; también pueden observarse pequeñas manchas de matorrales de encinos asociados con vegetación secundaria arbustiva que representa menos del 10\% de la superficie de la zona, mientras que la superficie restante se utiliza para la agricultura, El principal cultivo comercial es la caña de azúcar y su cosecha proporciona el mejor nivel de empleo para los productores de la región (Anónimo 2008).

Método. Entre los meses de mayo a octubre de 2000 a 2003, se efectuaron un total de 28 colectas esporádicas en la zona cañera del municipio de Chietla. Para obtener ejemplares adultos se realizaron colectas directas diurnas y nocturnas sobre follaje de arbustos y árboles. Asimismo se empleó una trampa de luz tipo pantalla, provista con una lámpara de luz de vapor de mercurio de 120 watts abastecida con un generador portátil de gasolina como fuente de energía, que se colocó en lugares abiertos rodeados por plantíos de caña de azúcar, la cual se mantuvo operando por periodos de dos a cinco horas dependiendo de las condiciones ambientales locales, por otro lado se revisaron los lugares con alumbrado público en donde son atraídos varios adultos de este grupo. Los ejemplares recolectados se colocaron en frascos letales con acetato de etilo; el $20 \%$ del material fue montado en alfiler entomológico y el resto se mantuvo en alcohol al 70\%. El material procesado se determinó a nivel de especie con ayuda de las claves dicotómicas para las faunas de Coleoptera Scarabaeoidea propuestas por Deloya \& Ratcliffe (1988), Morón (1986, 1994), Morón et al. (1988), Vaurie $(1958,1960)$, así como por comparación con las colecciones del Instituto de Ecología A. C. de Xalapa e Instituto de Ciencias de la Benemérita Universidad Autónoma de Puebla. Una vez determinado el material fue rotulado antes de ser depositado en la colección entomológica del Departamento de Agroecología y Ambiente del Instituto de Ciencias de la BUAP.

\section{RESULTADOS}

Se obtuvo información sobre un total de 1253 ejemplares representantes de cuatro familias, seis subfamilias, 11 tribus, 13 géneros y 23 especies de coleópteros lameli- 
cornios Cuadro 1. Se indican los datos biológicos y fenológicos de las especies, y en el apéndice I se incluye una clave dicotómica para su identificación.

\section{PASSALIDAE}

Ptichopus Macleay, 1869. En el mes de junio se registro $P$. angulatus a una altitud de $1180 \mathrm{~m}$ atraídos por la trampa de luz a $50 \mathrm{~m}$ de la zona agrícola.

\section{SCARABAEIDAE}

Digitonthophagus Balthasar, 1959. Se encontró a D. gazella durante el mes de junio atraído por la trampa de luz en zona agrícola.

Cuadro 1. Especies de coleópteros lamelicornios, colectados en la zona cañera de Atencingo, Puebla, México.

\begin{tabular}{|c|c|c|c|c|c|}
\hline Familia & Subfamilia & Tribu & Género & Especie & $\begin{array}{c}\mathrm{N}^{\circ} \mathrm{de} \\
\text { individuos }\end{array}$ \\
\hline Passalidae & Passalinae & Passalini & Ptichopus & angulatus & 3 \\
\hline Scarabaeidae & Scarabaeinae & Onthophagini & Digitonthophagus & gazella & 1 \\
\hline \multirow[t]{19}{*}{ Melolonthidae } & Melolonthinae & Diplotaxini & Diplotaxis & cribriceps & 16 \\
\hline & & & & megapleura & 7 \\
\hline & & Melolonthini & Polyphylla & concurrens & 1 \\
\hline & & & Phyllophaga & obsoleta) & 7 \\
\hline & & & & ravida & 59 \\
\hline & & & & setifera & 3 \\
\hline & & & & brevidens & 539 \\
\hline & & & & ilhuicaminai & 26 \\
\hline & Rutelinae & Anomalini & Paranomala & chevrolati & 2 \\
\hline & & & & cincta & 2 \\
\hline & & & & forreri & 2 \\
\hline & & & Epectinaspis & opacicollis & 1 \\
\hline & & Rutelini & Pelidnota & virescens & 1 \\
\hline & Dynastinae & Cyclocephalini & Cyclocephala & lunulata & 534 \\
\hline & & & & maffafa & 1 \\
\hline & & Pentodontini & Ligyrus & nasutus & 1 \\
\hline & & & & sallei & 19 \\
\hline & & Oryctini & Strategus & aloeus & 17 \\
\hline & & Phileurini & Phileurus & valgus & 1 \\
\hline \multirow[t]{2}{*}{ Cetoniidae } & Cetoniinae & Gymnetini & Cotinis & mutabilis & 9 \\
\hline & & & & pauperula & 1 \\
\hline Total & & & & & 1253 \\
\hline
\end{tabular}




\section{MELOLONTHIDAE}

Diplotaxis Kirby. Se obtuvieron registros de dos especies: D. cribriceps y D. megapleura. Ambas fueron colectadas en el mes de junio atraídos por la trampa de luz que se colocó a $5 \mathrm{~m}$ de la zona agrícola.

Polyphylla Harris, 1841. Representado por P. concurrens colectado durante la noche sobre vegetación a una altitud de $1180 \mathrm{~m}$.

Phyllophaga Harris, 1826. Se consiguieron cuatro especies del subgénero Phyllophaga y una del subgénero Phytalus. De $P$. ravida se colectaron durante el mes de junio en trampa de luz en zona agrícola, mientras que de $P$. setifera se colectaron durante la noche y no fueron atraídos por la luz mercurial, de $P$. brevidens fueron capturados 287 ejemplares en el mes de mayo en la trampa de luz, (12) durante el día sobre vegetación, en junio (213) los ejemplares fueron atraídos por la trampa de luz y 15 ejemplares fueron capturado en la luz fluorescente del alumbrado público y 12 individuos se recolectaron en julio en la trampa de luz. Mientras que $P$. ilhuicaminai se colecto en la trampa de luz durante el mes de junio y julio. En el caso de $P$. (Phytalus) obsoleta solo se colectaron dos machos y cinco hembras en el mes de junio atraídos por la trampa de luz.

Paranomala Casey, 1915. Se capturaron tres especies. De $P$. chevrolati y P. forreri se colectaron una pareja de cada una de ellas durante el mes de junio, la primera especie en la trampa de luz y la segunda en luz fluorescente del alumbrado público. $P$. cincta se encontraron en el mes de agosto atraídos por luz fluorescente.

Epectinaspis Blanchard, 1850. Durante septiembre un ejemplar de E. opacicollis fue atraído por la luz fluorescente del alumbrado público.

Pelidnota Macleay, 1819. Durante el día se colectó P. (Pelidnota) virescens en el mes de junio sobre vegetación silvestre a una altitud de $1150 \mathrm{~m}$ a $200 \mathrm{~m}$ de milpas y otros cultivos.

Cyclocephala Latreille, 1829. Se tienen registros de 534 ejemplares, de los cuales durante el mes de mayo se colectó una pareja de $C$. lunulata en el día sobre vegetación silvestre; en junio se recolectaron 371 ejemplares; en julio (122), agosto (15), octubre (26) todos en trampa de luz. Además se capturó C. maffafa en el mes de mayo atraído por la trampa de luz dentro de la zona agrícola.

Ligyrus Burmeister, 1847. Todos los ejemplares de este género fueron colectados con trampas de luz y bajo luz fluorescente, de bosque tropical mediano subcaducifolio, cultivos diversos y milpas. Se registró L. (Ligyrodes) sallei para los meses de junio (2), julio (9) y agosto (8) y L. (Ligyrus) nasutus en junio.

Strategus Hope, 1837. Únicamente se encontró $S$. aloeus durante junio (14) y julio (3). Se colectaron 13 individuos bajo lámparas de luz fluorescente del alumbrado público y en la trampa de luz mercurial, se registraron cuatro ejemplares.

Phileurus Latreille, 1807. De este género solo se encontró $P$. valgus durante agosto en la lámpara de luz fluorescente. 


\section{CETONIIDAE}

Cotinis Burmeister, 1842. Los ejemplares de dos especies de éste género se capturaron durante el día, sobre vegetación silvestre a una altitud de $1150 \mathrm{~m}$. De C. mutabilis se colectaron durante los meses de julio y agosto, y C. pauperula se capturó un ejemplar en septiembre.

\section{DISCUSIÓN}

El género mejor representado fue Phyllophaga con cinco especies, de las cuales $P$. brevidens y $P$. ravida fueron las más abundante con 539 y 59 ejemplares respectivamente, estos datos concuerdan por lo referido por Morón et al. 1996 quienes citan que para la zona cañera de Tepic, Nayarit el género Phyllophaga fue el más abundante y que a varias de sus especies se les atribuyen los daños al sistema radical de la caña de azúcar.

En el presente trabajo se encontró que una de la especie más abundante fue $C$. lunulata con 534 ejemplares, lo cual coincide con los registros de Aragón y Morón 2000, quienes mencionan que entre las larvas de melolóntidos encontrados en el sistema radical de la caña de azúcar de la zona de Atencingo, Puebla, la especie más abundante fue $C$. lunulata. En este trabajo se confirma su presencia y abundancia con muestras de adultos, sin embargo esta especie no es considerada como rizófaga estricta, porque sus larvas presentan hábitos saprófagos (Deloya 1998), por lo que los daños al cultivo de la caña de azúcar se atribuyen a $P$. brevidens y $P$. ravida. Con la información de este trabajo se complementa la información publicada por Aragón \& Morón (2000).

\section{CONCLUSIONES}

Se recolectaron 1253 ejemplares de 13 géneros y 23 especies de coleópteros lamelicornios, el género mejor representado fue Phyllophaga con cinco especies.

Las especies más abundantes fueron Cyclocephala lunulata y Phyllophaga brevidens con 534 y 539 ejemplares cada una; la primera puede ser una especie clave para el mejoramiento de los suelos de la región, y la segunda tiene importancia como plaga del sistema radical de la caña de azúcar, pero es necesario diseñar estudios de campo y laboratorio para confirmar ambas premisas y obtener más información agroecológica.

Agradecimientos. A la Red Nacional de Productividad y Calidad de Alimentos Agrícolas y al PROMEP, por el financiamiento otorgado para efectuar esta investigación.

\section{LITERATURA CITADA}

Anónimo. 2005. Enciclopedia de los municipios de México. Instituto Nacional para el Federalismo y el Desarrollo Municipal. Gobierno del Estado de Puebla, Puebla, México. 12 pp. 
Anónimo. 2008. Plan de Desarrollo Municipal de Chielta, Puebla 2008-2011. Orden jurídico Poblano. Puebla, Estado de Derecho y Justicia. Gobierno del estado de Puebla, Consejería Jurídica del Ejecutivo Estatal y Orden Jurídico Poblano, pp. 11-12.

Aragón, G. A. \& M. A. Morón. 1998. "Evaluación del daño ocasionado por el complejo "gallina ciega" (Coleoptera: Melolonthidae) en el estado de Puebla. pp. 143-149. In: Morón, M. A. \& A. Aragón G. (Eds.). Avances en el estudio de la diversidad, importancia y manejo de los coleópteros edafícolas americanos. Publicación especial de la Benemérita Universidad Autónoma de Puebla y la Sociedad Mexicana de Entomología, A. C. Puebla, México.

Aragón, G. A. \& M. A. Morón. 2000. Los coleópteros Melolonthidae asociados a la rizosfera de la caña de Azúcar en Chietla, Puebla, México. Folia Entomológica Mexicana, (108): 79-94.

Aragón, G. A., M. A. Morón, A. M. Tapia R., B. C. Pérez-Torres \& J. F. López-Olguín. 2010. Gallina ciega en Puebla, pp. 337-347. In: Rodríguez del Bosque L. A. \& M. A. Morón (Eds.). Plagas del Suelo. Mundi Prensa México. S.A. de C.V.

Deloya, C. 1998. Cyclocephala lunulata Burmeister, 1847 (Coleoptera: Melolonthidae, Dynastinae) asociada al cultivo de maíz (Zea mays) en Pueblo Nuevo, Morelos México, pp. 121-130. In: Morón M. A. \& A. Aragón G. (Eds.). Avances en el estudio de la diversidad, importancia y manejo de los coleópteros edaficolas americanos. Publicación especial de la Benemérita Universidad Autónoma de Puebla y la Sociedad Mexicana de Entomología, A. C. Puebla, México.

Deloya, C. \& B. C. Ratcliffe. 1988. Las especies de Cotinis Burmeister en México (Coleoptera: Melolonthidae: Cetoniinae). Acta Zoológica Mexicana (n.s.), 28: 1-52.

García, E. 1973. Modificación del sistema de clasificación climática de Köppen (Para adaptarlo a las condiciones de la República Mexicana) UNAM, México. 246 pp.

Morón, M. A. 1983, Introducción a la biosistemática y ecología de los coleópteros Melolonthidae edafícolas de México, pp. 1-13. In: II, Segunda Mesa Redonda sobre Plagas del Suelo, Sociedad Mexicana de Entomología, Colegio de Posgraduados y Depto. Parasitología, Universidad Autonóma de Chapingo, México.

Morón, M. A. 1986. El género Phyllophaga en México. Morfología, distribución y sistemática supraespecífica. (Insecta: Coleoptera). Publ. No 20 Instituto de Ecología. México, D. F. 341 pp.

Morón, M. A. 1994. Fauna de Coleoptera Lamellicornia en las montañas del noroeste de Hidalgo, México. Acta Zoológica Mexicana. (n. s.), (63):7-59.

Morón, M. A. 2003. Atlas de los escarabajos de México. Coleoptera: Lamellicornia. Volumen II, Familias Scarabaeidae, Trogidae, Passalidae y Lucanidae. Argania editio, Barcelona, España. 227 pp.

Morón, M. A. 2010. Diversidad y distribución del complejo "gallina ciega" (Coleoptera: Scarabaeoidea), pp. 51-76. In: Rodríguez del Bosque, L. A. \& M. A. Morón (Eds.). Plagas del suelo en México. MundiPrensa, México.

Morón, M. A., C. Deloya \& L. Delgado. 1988. Fauna de Coleópteros Melolonthidae, Scarabaeidae y Trogidae de la región de Chamela, Jalisco, México. Folia Entomológica Mexicana, (77): 313-378.

Morón, M. A., S. Hernández R. \& A. Ramírez C. 1996. El complejo "gallina ciega" (Coleoptera: Melolonthidae) asociado con la caña de azúcar en Nayarit, México. Folia Entomológica Mexicana, (98): 1-14.

Vaurie, P. 1958. A revision of the genus Diplotaxis (CoI. Scarab. Melolonthinae). Part I. Bulletin of the Americam Museum of Natural History, 1I5: 267-396.

Vaurie, P. 1960. A revision of the genus Diplotaxis (Col. Scarab. Melolonthinae). Part II. Bulletin of the Americam Museum of Natural History, 120: 161-434. 


\section{APÉNDICE I}

Clave para separar las especies de Coleoptera Lamellicornia de la zona cañera del municipio de Chietla, Puebla, México.

1 Antenas con el funículo recto. Maza antenal con tres a siete lamelas capaces de juntarse entre sí en reposo. Base de los élitros muy próxima a la base del pronoto $\ldots \ldots \ldots \ldots \ldots \ldots \ldots \ldots 2$

1' Antenas con el funículo capaz de doblarse en forma de "C". Maza antenal con cinco lamelas separadas entre sí. Base de los élitros claramente separada de la base del pronoto. Mandíbulas con dos dientes apicales, uno grande y otro pequeño. Long. 27-33 mm . . . . . Ptichopus angulatus (Perch.)

2 Maza antenal brillante, con sedas conspicuas y escasas. Estigmas abdominales colocados sobre los extremos laterales de los últimos tres esternitos, de tal forma que es posible observar de uno a tres

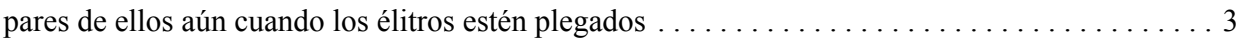

2' Maza antenal opaca, cubierta de sedas microscópicas. Estigmas abdominales colocados sobre los pleuritos, de tal forma que no es posible observar ninguno de ellos cuando los élitros están plegados Pronoto con el margen laterobasal pardo amarillento y el disco negro. Élitros pardo amarillentos. Machos con dos proyecciones ceratiformes recurvadas en la frente y con una quilla frontoclipeal. Longitud corporal 9-11 mm ................ Digitonthophagus gazella (Fabricius)

3 Mesopímeros visibles dorsalmente. Escotadura anteocular amplia, permite ver la inserción de la antena desde el dorso. . . . . CETONIIDAE. . . . . Escutelo cubierto por el lóbulo basal del prono-

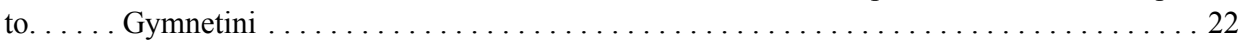

3' Mesopímeros ocultos. Escotadura anteocular no existe, no es posible ver la inserción de la antena

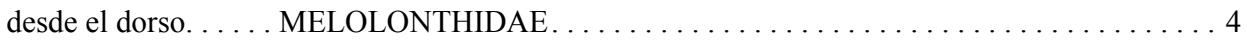

4 Borde anterior del labro completamente oculto bajo el clípeo. Uñas de los meso y metatarsos sencillas. . . Dynastinae. . . . . . . . . . . . . . . . . . . . 17

4' Borde anterior del labro más o menos visible frente y debajo del clípeo. Uñas meso y metatarsales

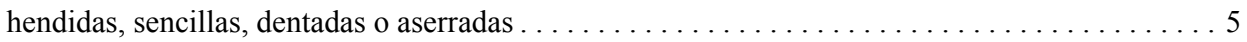

5 Uñas tarsales retráctiles, las dos uñas de cada pata tienen diferente anchura, grosor, forma o longitud. Mandíbulas usualmente visibles en parte a los lados del clípeo. . . . Rutelinae . . . . . 13

5' Uñas tarsales no retráctiles, en general las dos uñas de cada pata tienen la misma forma, y son hendidas, dentadas o aserradas. . . . Melolonthinae . . . . . . . . . . . . . . . . 6

6 Procoxas estrechas, prominentes, semicónicas. Esternito VI muy reducido, poco notable. Placa pi-

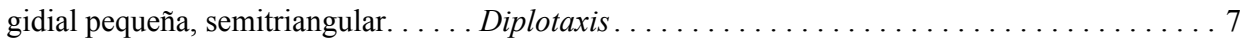

6' Procoxas anchas, transversales, poco prominentes. Esternito VI notable, con amplitud variable. Placa pigidial ancha, ovalada, usualmente convexa y prominente $\ldots \ldots \ldots \ldots \ldots \ldots \ldots$

7 Márgenes laterales del clípeo ligeramente sinuados, de forma trapezoide. Placa pigidial ovalada con punteado profundo. Margen apical de los parámeros redondeados. Longitud corporal 0.8-0.9

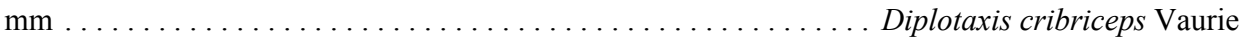

7' Márgenes laterales del clípeo ligeramente dentiformes. Placa pigidial densamente punteada. Margen interno de los parámeros articulados en su cuarto o quinto basal. Longitud corporal 6.5-8.5

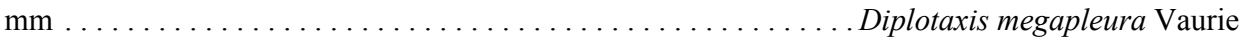

8 Maza antenal con siete laminillas muy largas en machos y con cinco laminillas cortas en hembras. Pronoto y élitros con franjas longitudinales de escamas blancas. Tegumento dorsal pardo rojizo, con los bordes de las franjas elitrales blancas irregulares. Longitud corporal 32.0-38.0

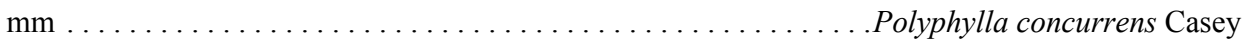

8' Maza antenal con tres laminillas cortas o largas en machos y hembras. Pronoto y élitros con vestidura variable, longitud corporal inferior a $25.0 \mathrm{~mm} \ldots \ldots$ Phyllophaga . . . . . . . . 9 
9 Uñas bífidas o con una proyección dentiforme cercana al ápice. Dorso glabro. Quinto esternito abdominal con una proyección laminar media semitriangular cóncava cubierta con sedas y sus bordes distales finamente pectinados. Parámeros cortos, estrechos y fusionados en sus extremos apicales . . . . . . . . . . . . . . . . . . Phyllophaga (Phytalus) obsoleta (Blanchard)

9' Uñas con un proceso dentiforme en el borde inferior, alejado del ápice. Quinto esternito y parámeros con forma diversa..... Phyllophaga (Phyllophaga) .................. 10

10 Pronoto y élitros brillantes, glabros o con algunas sedas aisladas $\ldots \ldots \ldots \ldots \ldots \ldots \ldots \ldots 11$

$10^{\prime}$ Pronoto y élitros opacos o pruinosos, cubiertos con abundantes sedas cortas y algunas sedas más

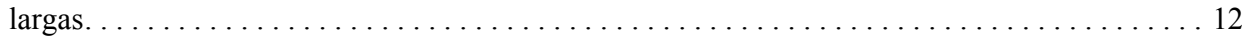

11 Uñas mesotarsales externas de machos con los dentículos asimétricos. Parámeros cortos, fusionados entre sí, con el ápice laminar recto. Longitud corporal 13.5 a $20.0 \mathrm{~mm}$. . . . . . Phyllophaga (Phyllophaga) ravida (Blanchard)

11' Todas las uñas tarsales simétricas, con un dentículo pequeño en el borde inferior, cercano a la base unguinal, parte del borde inferior finamente aserrado. Longitud corporal 14.0-18.0

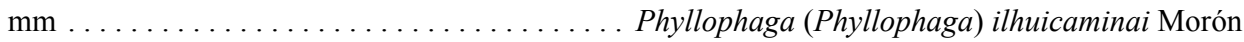

12 Protarsómeros 1-3 con proyecciones laminares ventroapicales. Parámeros cortos, anchos, fusionados en la base y ápice, con dentículos laterales largos y afilados. Longitud corporal 13.9-16.0

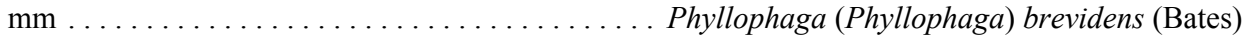

12' Protarsómeros 1-3 sin proyecciones laminares ventroapicales. Parámeros cortos, anchos, fusionados en su base y ápice, con dentículos laterales cortos. Longitud corporal 15.0-19.0 $\mathrm{mm} \ldots \ldots \ldots \ldots \ldots \ldots \ldots \ldots \ldots \ldots \ldots \ldots \ldots \ldots$ Phyllophaga (Phyllophaga) setifera (Burmeister)

13 Orilla exterior de los élitros con un borde membranoso. . . . Anomalini . . . . . . . . . . 14

13' Orilla exterior de los élitros sin borde membranoso. . . . . Rutelini. . . . . Región dorsal testácea, iridiscente, amarillenta, blanquecina, nacarada muy brillante. Regiones ventrales verde metálico. Longitud corporal 18.0-25.0 mm ............. Pelidnota (Pelidnota) virescens Burmeister

14 Mesoepímeros ligeramente sobresalientes. Superficie del pronoto cubierta con abundantes sedas cortas. Élitros con los bordes laterales sinuados. Placa pigidial con sedas largas . . . . Epectinaspis opacicollis Bates

14' Mesoepímeros ocultos por completo. Superficie del pronoto glabra o con escasas sedas aisladas. Élitros con los bordes laterales rectos o curvados. Placa pigidial glabra o con sedas cor-

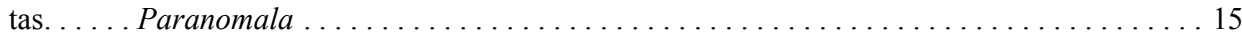

15 Pronoto con una mancha obscura grande que no alcanza el borde posterior. Élitros amarillo testáceo, solo con los bordes suturales obscuros. Longitud corporal 14.0-15.0 mm . . . . . Paranomala chevrolati Bates

15' Pronoto verde obscuro con reflejos verde metálico intenso. Elitros pardo amarillento uniforme o

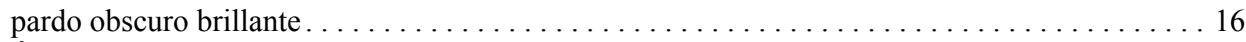

16 Élitros pardo amarillento con puntos diminutos. Borde exterior de las protibias con dos dientes claramente marcados y un tercer dentículo proximal mucho menos acentuado que los precedentes.

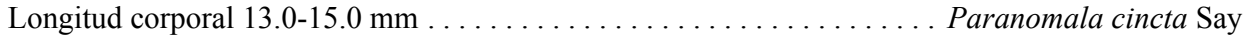

$16^{\prime}$ Élitros pardo obscuro finamente rugosos. Borde exterior de las protibias con dos dentículos el segundo mucho más corto que el primero. Longitud corporal 14.0-16.0 mm. . . Paranomala forreri Bates

17 Élitros poco convexos, con estrías y punteado profundo. Borde exterior de las protibias con cuatro dentículos afilados. Base de la placa pigidial con un surco transversal profundo. Longitud corporal

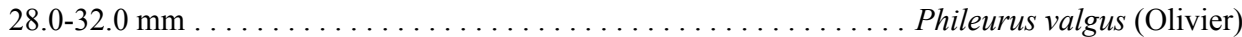

17' Élitros convexos, casi lisos o con puntos y estrías someras. Borde exterior de las protibias con 3 o 4 dentículos redondeados o aguzados. Base de la placa pigidial $\sin$ surco transversal . . . . . 18 
18 Cabeza y pronoto con carinas, tubérculos, depresiones o procesos ceratiformes $\ldots \ldots \ldots \ldots 20$

18' Cabeza y pronoto sin carinas, tubérculos o prominencias notorias. . . . Cyclocephalini. ..... Clípeo semitrapezoidal con puntuación fina y regular. Espolones metatibiales estrechos y agu-

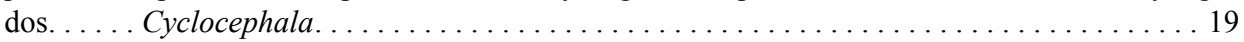

19 Clípeo testáceo. Frente pardo obscuro. Pronoto con tres conjuntos de franjas o manchas irregulares poco definidas. Cada élitro con dos conjuntos de franjas parduzcas transversales, irregulares, sinuosas o angulosas muy variables en extensión e intensidad. Longitud corporal 13.0-16.0

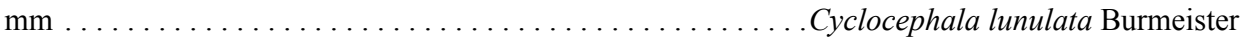

19' Clípeo y frente pardo obscuro. Pronoto con dos franjas sinuosas longitudinales negras. Cada élitro con cuatro a seis manchas obscuras irregulares y el borde sutural obscuro. Esternitos y placa pigidial pardo obscura casi negro. Longitud corporal 21.0-25.0 mm . . . Cyclocephala maffafa Burmeister

20 Ápice de las tibias posteriores casi recto con numerosas sedas cortas espiniformes. Pronoto en los dos sexos convexo. Color variable entre pardo oscuro y pardo rojizo brillante. . . . . Ligyrus . . 21

$20^{\prime}$ Ápice de las tibias posteriores con dentículos grandes y escasas sedas cortas. Pronoto con una excavación amplia y profunda, en las hembras rodeada por tres tubérculos redondeados y en los machos con un tubérculo comprimido a cada lado y una proyección ceratiforme al frente. Cabeza y pronoto pardo obscuro, élitros, abdomen y patas pardo rojizo brillante. Longitud corporal 28.0-50.0

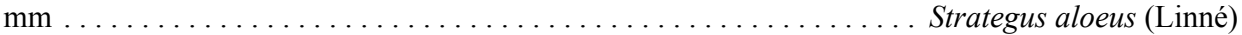

21 Borde extremo de las protibias con tres dentículos grandes y dos dentículos pequeños intercalados entre los grandes. Ápice del clípeo truncado. Quilla fronto-clipeal vaga. Cuerpo pardo obscuro brillante. Longitud corporal 18.0-21.0 mm............... Ligyrus (Ligyrodes) sallei Bates

$21^{\prime}$ Borde extremo de las protibias con tres dentículos grandes, sin dentículos pequeños intercalados. Ápice del clípeo un poco aguzado. Con un quilla fronto-clipeal bien desarrollada. Cuerpo pardo obscuro o rojizo brillante. Longitud corporal 17.0-19.0 mm . . . . . . . . Ligyrus (Ligyrus) nasutus Burmeister

22 Frente con una proyección notable en su línea media, dirigida hacia la frente y separada de la superficie frontal. Cabeza y región ventral verde metálico intenso. Pronoto y élitros opacos finamente pruinosos, verde oliva a verde hierba, en ocasiones con franjas contrastantes amarillentas o anaranjadas. Longitud corporal 21.0-30.0 mm ............ Cotinis mutabilis (Gory y Percheron)

$22^{\prime}$ Frente con un reborde longitudinal. Cabeza, élitros y región ventral verde obscuro. Pronoto verde opaco. Longitud corporal 21.0-23.0 $\mathrm{mm}$. . . . . . . . . . Cotinis pauperula (Burmeister) 